\section{Incorporación del enfoque intercultural en el sistema de salud peruano: la atención del parto vertical}

\author{
César R. Nureña ${ }^{1}$
}

Forma de citar: Nureña CR. Incorporación del enfoque intercultural en el sistema de salud peruano: la atención del parto vertical. Rev Panam Salud Publica. 2009;26(4):368-76.

Palabras clave: diversidad cultural; parto; salud indígena; política de salud; Perú.

\footnotetext{
Universidad Nacional Mayor de San Marcos, Facultad de Ciencias Sociales, Escuela de Antropología. La correspondencia debe dirigirse a César Nureña, Escuela de Antropología, Facultad de Ciencias Sociales, Universidad Nacional Mayor de San Marcos, Av. Venezuela, cuadra 34, Lima 1, Perú. Correo electrónico: cnurena@yahoo.es
}

El Perú alberga muchas culturas que guardan entre sí relaciones muy diversas, a menudo conflictivas, pero que en ocasiones también revelan puntos de convergencia y aprendizajes recíprocos. El Estado nacional se define a sí mismo como democrático y representativo de esa pluralidad de culturas, pero con un sello propio que expresa pautas y valores hegemónicos sobre otras culturas escasamente reconocidas en él. Estas tensiones responden a relaciones de poder y asimetrías sociales y económicas. Es así como los grupos mejor posicionados respecto del aparato estatal logran propagar sus valores y modos de vida a la sociedad en su conjunto, en particular, a la forma de gobierno, el sistema educativo, la lengua, la administración de justicia, y las prácticas médicas. Puede ocurrir, sin embargo, que algunos elementos de las culturas no hegemónicas lleguen a formar parte de la comunidad nacional más amplia, que ciertos sectores sociales incorporen recursos culturales de otros, o que haya grupos que se resistan a adoptar nuevas ideas o prácticas.

Si bien predomina en el país una matriz cultural hispano-occidental en la mayor parte del territorio, existen grupos que mantienen formas de vida y visiones del mundo particulares y diferentes. Estas diferencias se ponen de manifiesto también en el conjunto de prácticas y conocimientos relacionados con la salud, la enfermedad y el bienestar físico y emocional. Se observan así tensiones culturales entre la "medicina moderna" occidental y la medicina indígena. ¿Cómo abordar estas cuestiones? ¿Es posible conciliar estas prácticas? Lo que suele ocurrir es que se acepta sin más la imposición de la medicina occidental, dejando de lado las prácticas alternativas. Se pierde entonces parte del conocimiento y las tradiciones acumulados durante miles de años. No obstante, junto al creciente reconocimiento de la diversidad de culturas y de los derechos sociales y culturales de los pueblos indígenas, han surgido en los últimos tiempos propuestas de políticas públicas y se han establecido organismos estatales orientados a efectivizar en la práctica el ejercicio de tales derechos. En el Ministerio de Salud del Perú ya existen instancias encargadas de las políticas y programas de salud de los indígenas, algunas de las cuales han intervenido en la incorporación de la atención del parto en posición vertical "con adecuación cultural".

En este trabajo se revisan los antecedentes, las políticas y las normas relativos a la institucionalización del parto vertical en el país. Se han consultado para ello fuentes oficiales, informes de organismos internacionales y otros documentos, con una perspectiva crítica y teniendo en cuenta conceptos y enfoques sobre el proyecto de interculturalidad. 


\section{SITUACIÓN SOCIOECONÓMICA Y DE SALUD DE LOS PUEBLOS INDÍGENAS}

Según datos oficiales, existen en el Perú alrededor de 72 grupos étnicos (65 en la Amazonía, y los siete restantes principalmente en la región andina), clasificados en 14 familias etnolingüísticas; en su mayoría, esos grupos están organizados como comunidades campesinas (5 069 en el área andina) y comunidades nativas (961 en la Amazonía) $(1,2)$. Hay también unos pocos pueblos indígenas que los organismos oficiales consideran en situación de "aislamiento voluntario" o de "contacto inicial". Desde luego, las fronteras entre las culturas son siempre porosas y dinámicas, por lo que muchas veces resulta difícil determinar quién es indígena y quién no lo es. ${ }^{2}$ Se estima que la población indígena en el Perú representa alrededor de $40 \%$ del total aproximado de 28 millones de habitantes. ${ }^{3}$

El Perú es uno de los países de las Américas con más proporción de población indígena y mayor diversidad étnica. A pesar de que los conocimientos colectivos de los pueblos indígenas expresan gran parte de la riqueza del patrimonio cultural del Perú, sus comunidades se encuentran en situación desventajosa en relación con los mestizos y con otros grupos más ligados a la cultura dominante. Las profundas desigualdades e inequidades políticas y económicas se manifiestan con particular dureza en aspectos como la situación de salud y las demás condiciones de vida y bienestar. Los objetivos nacionales de desarrollo y las estrategias de reducción de la pobreza no tienen en cuenta en la práctica la identidad, la cultura y la cosmovisión de los pueblos indígenas, su derecho a la autodeterminación y a controlar sus territorios y recursos, ni tampoco una concepción integral de la salud. La incidencia de la pobreza es desproporcionadamente mayor entre los grupos indígenas en comparación con los no indígenas, y va acompañada de otros factores adversos, como la discriminación, el analfabetismo, la falta de acceso a los servicios sociales, las violaciones de derechos, los conflictos armados, el narcotráfico y el deterioro ambiental (3).

La población indígena presenta tasas muy elevadas de mortalidad materna e infantil, malnutrición y enfermedades infecciosas. Además, con el aumento de la migración indígena a las ciudades, nuevos factores pueden agravar sus condiciones de salud. Aunque los índices de morbilidad y el perfil epidemiológico de los aborígenes sean similares a los de otros grupos desfavorecidos, su situación desventajosa se ve exacerbada a causa de los prejuicios y la discriminación de origen étnico ejercida muchas veces por el propio personal de

\footnotetext{
El Convenio 169 de la Organización Internacional del Trabajo (OIT) define pueblos indígenas como: "[. . .] los pueblos en países independientes, considerados indígenas por el hecho de descender de poblaciones que habitaban en el país o en una región geográfica a la que pertenece el país en la época de la conquista o la colonización o del establecimiento de las actuales fronteras estatales y que, cualquiera que sea su situación jurídica, conservan todas sus propias instituciones sociales, económicas, culturales y políticas, o parte de ellas".

3 Según datos de la Organización Panamericana de la Salud (OPS), en 1993 esa proporción era de $47 \%$.
}

salud. La prestación de servicios de salud de baja calidad afecta también a otros sectores de la población nacional, pero alcanza los peores niveles en las zonas habitadas por los pueblos indígenas. El personal de salud posee en general una limitada competencia en materia intercultural, y es difícil retenerlo en las zonas rurales. Por otra parte, aunque la asistencia sanitaria suele ser gratuita para las comunidades campesinas y nativas, las barreras geográficas dificultan el acceso a los servicios, cuyos costos incluyen, en un análisis riguroso, gastos de transporte, alimentos, alojamiento y medicamentos, así como desamparo familiar y pérdida de días laborables. A ello se añaden deficiencias en el suministro de medicamentos y en el equipamiento, sobre todo en las zonas de más difícil acceso.

Las barreras culturales plantean un reto mucho más complejo, pues persiste una escasa comprensión de los conocimientos y las prácticas de salud tradicionales, si bien se han logrado algunos avances esporádicos. El sesgo a favor de las intervenciones de salud occidentales suele resultar ofensivo o impropio para quienes recurren habitualmente a la medicina tradicional. Las dificultades del sistema de salud para encontrar profesionales que hablen lenguas indígenas complican aún más la situación. Las barreras comunicacionales entre los prestadores de asistencia sanitaria y la población indígena limitan el acceso a una atención de calidad. Por otro lado, el temor y la desconfianza hacia los trabajadores de salud, causados muchas veces por prejuicios, discriminación o desencuentros culturales, impiden a menudo que los indígenas busquen en el sistema de salud la atención que necesitan. Por ejemplo, un informe reciente (4) sobre la salud de los pueblos indígenas en el Perú revela un escenario de carencias crónicas en el financiamiento de los programas y servicios de salud, a lo que se suma la poca o nula capacitación del personal en aspectos relacionados con las culturas locales, y el hecho de que los médicos destacados en zonas rurales, sin incentivos y frente a duras condiciones de trabajo, desean abandonarlas lo antes posible.

En lo que respecta a las políticas, la escasez de información y de estadísticas sobre los pueblos indígenas dificulta los procesos de gestión, la definición de prioridades, la creación de políticas y el establecimiento de sistemas de evaluación apropiados. Tampoco hay una participación indígena uniforme en la elaboración e implementación de iniciativas. Afortunadamente, se aprecian en la actualidad algunos avances, como los estudios que está realizando el Ministerio de Salud sobre algunas etnias amazónicas $(5,6)$. Sin embargo, se han desatendido cuestiones tales como el cuidado del medio ambiente, la protección de la diversidad biológica, las patentes sobre principios activos de plantas, y los derechos de propiedad colectivos.

Entre 1997 y 1998, un equipo del Ministerio de Salud realizó visitas a 18 comunidades nativas y de colonos mestizos en Junín y Ucayali. El informe correspondiente (1) reveló índices elevados de enfermedades respiratorias, tuberculosis, parasitosis y enfermedades diarreicas agudas (vinculadas a la contaminación de las 
aguas de los ríos), cuadros de malnutrición y falta de acceso a medicamentos. Para empeorar la situación, muchas comunidades afrontan la violencia derivada de las actividades de grupos armados y narcotraficantes. El Ministerio de Salud es el principal proveedor de asistencia sanitaria para los indígenas. Algunas organizaciones no gubernamentales (ONG) han ejecutado programas de capacitación de promotores de salud y de parteros y parteras tradicionales; asimismo, han colaborado en la instalación de botiquines comunales, aunque con problemas en el suministro de medicamentos.

La tasa global de mortalidad materna en el país es de 164,2 defunciones maternas por 100000 nacidos vivos (2002). Esta cifra nacional ya es de por sí bastante alta, pero en las zonas donde la población mayoritaria está compuesta por indígenas la tasa se eleva notoriamente. Con respecto a la mortalidad infantil, si bien se ha notificado una notable reducción de muertes neonatales en las zonas rurales (de 34 a 16 defunciones por 1000 nacidos vivos), aún sigue habiendo marcadas diferencias con las zonas urbanas (7). Lamentablemente, los indicadores generados por los sistemas de información sanitaria muestran solo diferencias por departamentos, edad, sexo y lugar de residencia (rural/urbano), entre otros, pero no incluyen variables como pertenencia étnica o lengua.

\section{EL ENFOQUE DE SALUD INTERCULTURAL EN LA ORGANIZACIÓN PANAMERICANA DE LA SALUD}

Durante los decenios de 1980 y 1990, en el marco del proceso de globalización y sus repercusiones, han surgido y se han consolidado nuevos movimientos sociales. A diferencia de los anteriores, basados principalmente en criterios de adscripción política, económicos o de clase (por ejemplo, partidos de izquierda, centro o derecha, agrupaciones obreras o campesinas, etc.), estas nuevas corrientes encuentran su fundamento en reivindicaciones culturales, étnicas, de género y de orientación sexual, entre otras. En los países de la Región y en los foros internacionales, comienzan también a hacer sentir su presencia los movimientos y organizaciones indígenas.

Durante este período cobran fuerza formulaciones políticas y académicas que plantean un proyecto de interculturalidad en las relaciones entre pueblos étnicamente diversos. En 1992, la OPS propone que se considere más detenidamente la salud y el bienestar de los indígenas. En 1993 se organizó una reunión en Winnipeg, Canadá, que congregó a representantes de los pueblos indígenas, de los gobiernos y otros interesados de 18 países de la Región. Las recomendaciones del encuentro, agrupadas en la propuesta "Iniciativa de salud de los pueblos indígenas", planteaban: i) la necesidad de considerar la salud con un criterio integral; ii) el derecho de los pueblos indígenas a la autodeterminación y a la participación sistemática; iii) el respeto por las culturas indígenas y su revitalización; y iv) la reciprocidad en las relaciones con otros grupos. En adelante, la mayor parte de los países de la Región tuvieron más en cuenta estas orientaciones en sus sistemas de salud. Se empezaron a considerar las demandas de los grupos indígenas de cada país y se planificaron acciones más acordes con las propuestas de organismos de cooperación técnica como la OPS, cuya Iniciativa se integró en el "Decenio Internacional de las Poblaciones Indígenas del Mundo" (1994-2004) promovido por las Naciones Unidas. Las actividades planteadas en los planes de acción fueron evaluadas al finalizar este período.

Una reunión de expertos de 19 países, realizada en Panamá en 2005, sirvió para mostrar una serie de logros y retos en materia de salud indígena (3). Se supo entonces que la mayoría de las naciones han aprobado actualmente al menos una directiva legal que sirve como marco de política sobre los pueblos indígenas, aunque ninguna tiene en cuenta las disparidades sociales y económicas. Muchos países han ejecutado políticas o reproducido experiencias que promueven la incorporación de los tratamientos y las medicinas tradicionales en sus respectivos sistemas sanitarios, y otros han dispuesto mediante proyectos específicos una observación intercultural más profunda de las prácticas de salud tradicionales. Casi todos cuentan ahora con unidades técnicas especializadas dedicadas a los asuntos indígenas en sus ministerios de salud, pero muchas veces esas oficinas no cuentan con el apoyo político, el personal y el presupuesto necesarios para realizar sus funciones en forma eficaz. Aunque se han realizado algunos estudios y estimaciones, no hay una caracterización demográfica clara de los indígenas, ni un sistema fiable de información y evaluación de sus condiciones de salud; y muy pocos países cuentan con programas de investigación específicos. Persisten, además, diversos obstáculos que dificultan el cumplimiento de los objetivos planteados, tales como la insuficiente capacitación intercultural de los trabajadores de salud y de los decisores políticos; la escasa participación de las comunidades en la definición de políticas y prioridades; la pobreza, la falta de acceso a la tierra, y otros problemas socioeconómicos. Por otra parte, la creciente presión de las organizaciones indígenas ha determinado su mayor visibilidad y ha posibilitado algunos avances en cuanto a la participación política en el interior de las comunidades nacionales.

Con base en estas evaluaciones, la OPS propuso como líneas de acción incorporar la perspectiva indígena en los Objetivos de Desarrollo del Milenio $(\mathrm{ODM})^{4}$ y en las políticas nacionales de salud, mejorar la gestión del conocimiento e integrar el abordaje intercultural en los sistemas de salud. Algunas de las actividades planteadas son fomentar el diálogo entre las autoridades, los indígenas y la sociedad civil, e incorporar variables relativas a la etnicidad en los sistemas

\footnotetext{
4 Los ODM constituyen un plan acordado entre los líderes de 189 estados (2000), que establece metas y plazos para combatir la pobreza, el hambre, las enfermedades, el analfabetismo, la degradación del medio ambiente y la discriminación contra la mujer, en consonancia con los principios de los derechos humanos, el gobierno responsable y la democracia.
} 
de información. Se contempla también la capacitación en materia de desigualdad y discriminación, además de la promoción de investigaciones sobre la armonización del conocimiento indígena y el occidental. La OPS considera que la incorporación del enfoque intercultural puede efectivizarse mediante sistemas de autorización y acreditación con criterios de interculturalidad, la elaboración de protocolos para intervenciones culturalmente apropiadas, y el desarrollo de modelos de buenas prácticas interculturales de atención.

\section{EL ENFOQUE INTERCULTURAL EN LAS POLITICAS SANITARIAS DEL PERÚ}

El Ministerio de Salud del Perú reorganizó sus programas en el año 2004 y definió diez Estrategias Sanitarias Nacionales (ESN). Se estableció una ESN de Salud de los Pueblos Indígenas, y otra de Salud Sexual y Reproductiva, esta última directamente relacionada con la atención del parto vertical. El Centro Nacional de Salud Intercultural (CENSI), dependiente del Instituto Nacional de Salud (INS, organismo del Ministerio de Salud especializado en investigación) es responsable de la ESN de Salud de los Pueblos Indígenas. Su función es promover, coordinar y supervisar las acciones dirigidas a las poblaciones indígenas, con el propósito de disminuir la brecha de exclusión en salud. Hasta hace algunos años, existía el Instituto Nacional de Medicina Tradicional (INMETRA), cuyas funciones, consistentes en investigar, rescatar y revalorizar los conocimientos y experiencias tradicionales de las diversas etnias del país, se integraron posteriormente al INS. De acuerdo con la ESN de Salud de los Pueblos Indígenas, se ha constituido una Comisión Nacional de Salud de los Pueblos Indígenas y, según la Memoria 2001-2006 del Ministerio, se está trabajando en la adecuación cultural y la ampliación de la oferta de servicios bajo nuevas modalidades apropiadas para los pueblos indígenas; el "respeto al parto vertical"; la capacitación de los recursos humanos en interculturalidad; el fortalecimiento de la participación comunitaria, y el mejoramiento del sistema de vigilancia epidemiológica. El programa destaca entre sus actividades: el desarrollo de diseños para un censo de comunidades indígenas; la aprobación de una norma para el aseguramiento universal de la población dispersa y extremadamente pobre, incluidos los pueblos indígenas; el desarrollo de categorías étnicas a ser incorporadas en los sistemas de información, y la elaboración de un plan para la protección de comunidades indígenas en aislamiento voluntario y en contacto inicial.

Por su parte, también la ESN de Salud Sexual y Reproductiva ha introducido el enfoque intercultural en sus orientaciones programáticas. Propone, entre otras cosas, mejorar la calidad de la atención del parto y del recién nacido, la adecuación cultural de los servicios, el desarrollo de actividades de educación, y la prestación de servicios teniendo en cuenta "una perspectiva de género, derechos humanos e interculturalidad". Hace hincapié en la disminución de las tasas de mortalidad materna y perinatal y en el incremento de la atención del parto en instituciones de salud, procurando reducir las barreras económicas (mediante el seguro integral de salud), y también las geográficas (mediante las casas de espera) y culturales. Ha elaborado asimismo una norma técnica para la atención del parto vertical (8).

Otro organismo del Ministerio que trabaja estas cuestiones es la Unidad Técnica Funcional de Derechos Humanos, Equidad de Género e Interculturalidad en Salud, creada en 2005 y adscrita a la Dirección General de Promoción de la Salud (DGPS). Cuando se preparaba este trabajo se publicó una nueva norma, promovida por esa oficina, para la "transversalización" de los enfoques de derechos humanos, equidad de género e interculturalidad en los programas y departamentos del Ministerio. Finalmente, en la Dirección General de Epidemiología (DGE) del Ministerio de Salud se establecieron un Grupo Temático de Salud de los Pueblos Indígenas, y una Unidad Técnica de Proyectos Especiales en Pueblos Indígenas, que han realizado una serie de estudios llamados Análisis de la Situación de Salud (ASIS) en etnias específicas de la Amazonía, con la participación de las comunidades $(5,6)$.

\section{ADECUACIÓN CULTURAL: CASAS DE ESPERA Y PARTO VERTICAL}

El parto en diversas variantes de la posición vertical constituye una práctica milenaria de quizá todas las culturas, como puede apreciarse en la iconografía antigua. En Europa occidental, en el siglo XVII comenzó la práctica de "acostar" a las mujeres para parir, probablemente para facilitar el uso del fórceps, instrumento que comenzó a usarse para ayudar en el alumbramiento. Con la introducción y difusión en el siglo XX de la anestesia peridural durante el trabajo de parto, se fue imponiendo cada vez más la posición horizontal. Estos y otros procedimientos se inscriben dentro del enfoque biomédico occidental. Pero mientras que en este enfoque se entiende el parto como una condición de salud que requiere intervención médica, en otros contextos se lo considera un hecho natural (9). Hoy el parto vertical se está difundiendo en muchos países, y esto ha impulsado un debate académico sobre las ventajas y desventajas de una u otra modalidad de parto.

En el sistema de salud peruano, la atención del parto en posición vertical se realiza formalmente desde comienzos del decenio de 1990, aunque ya mucho antes se practicaba en el ámbito domiciliario. Hacia fines de ese decenio, el Proyecto 2000, una importante iniciativa de salud maternoinfantil, emprendió diversos estudios e intervenciones para evaluar e impulsar la atención del parto vertical $(9,10)$. En 2005, el Ministerio de Salud publicó la norma técnica (8) que establece el marco procedimental para la atención del parto vertical. Además, el sistema de salud ha implementado y difundido en diversas zonas algunas innovaciones que consideran la introducción de prácticas tradicionales de las culturas locales, y formas de participación de la comunidad, tales como el "acompañamiento" y las casas de espera. 
El acompañamiento se realiza a veces en lugares donde las distancias entre los servicios de salud y el domicilio de las gestantes son muy grandes; en tales casos, el personal de salud puede, por ejemplo, ir a buscar a las mujeres "hasta la mitad del trayecto". En otras ocasiones, los municipios $\mathrm{u}$ otros organismos costean el transporte. La segunda modalidad participativa, las casas de espera, integra una estrategia que incluye la intervención activa tanto de las autoridades sanitarias como de las organizaciones locales. Algunas de estas casas se autofinancian, mientras que otras funcionan con el apoyo del establecimiento de salud o de los municipios. La historia de las casas de espera comenzó en 1998 en la ciudad de Huánuco, donde se instalaron las dos primeras coordinadas por el Ministerio de Salud y el Fondo de las Naciones Unidas para la Infancia (UNICEF); con ellas se buscaba crear espacios comunitarios cercanos a los establecimientos de salud, que sirvieran para alojar a las gestantes durante los días previos al alumbramiento y atender posibles complicaciones, como una forma de ayudar a reducir la mortalidad materna. Las casas fueron incorporando elementos de la partería tradicional andina, como el parto vertical, la climatización del ambiente, las mantas de cuero, la preparación de mates o infusiones con distintas hierbas, y el acompañamiento de familiares $\mathrm{u}$ otros allegados. Con posterioridad a esa experiencia se ha señalado que el propósito de las casas de espera no es que la mujer diera a luz allí, sino que se la prepare para tener el parto en un establecimiento de salud cercano (11). Al principio sí hubo algunos partos en las casas de espera atendidos por parteras tradicionales, pero paulatinamente se fue difundiendo la atención del parto por profesionales en establecimientos de salud, cuyas prácticas y servicios tuvieron también que ir adaptándose a las necesidades y expectativas de la población teniendo en cuenta el enfoque de adecuación cultural. En las casas de espera las mujeres pueden seguir desarrollando muchas de sus actividades cotidianas, como lavar, cocinar o atender a su familia, y en algunos casos se organizaron talleres productivos (de manualidades o artesanía, por ejemplo). Informes oficiales estiman que en el país funcionan unas 337 casas de espera.

Los promotores de salud o agentes comunitarios de salud, que a veces son también parteros o parteras, desempeñan un papel importante al actuar como nexos entre la población y el sistema sanitario, y participan en forma activa en la atención de las gestantes. Reciben capacitación del Ministerio, y ellos a su vez ayudan al personal de salud a acercarse a la gente, para adecuar culturalmente la atención sanitaria y crear relaciones de confianza entre los servicios de salud y la población.

De acuerdo con la norma técnica, la atención del parto vertical debe incorporar elementos y prácticas de adecuación intercultural integrando saberes y procedimientos tradicionales en el plan de parto, la organización de los recursos, las intervenciones requeridas antes, durante y después del alumbramiento, el ma- nejo de las complicaciones y también en la consejería. El plan de parto debe consignar las preferencias de la mujer en relación con el lugar del alumbramiento y el transporte disponible, la posición elegida para el parto y las personas que la acompañarán. La adecuación intercultural se refiere al acondicionamiento del local (iluminación tenue, calefacción, cortinas, etc.), la presencia de parteras y de allegados, y la disponibilidad de materiales y equipos (vestimenta, fajas, camillas, etc.) y de medicinas tradicionales. Hay recomendaciones específicas relacionadas con las revisiones físicas, en particular el examen vaginal, así como con el manejo del dolor, los masajes, la relajación y la higiene. La parturienta podrá optar por alguna de las posiciones siguientes: en cuclillas, de rodillas, sentada o semisentada, tomada de una soga o apoyada en manos y pies. Los familiares o las parteras tradicionales también pueden participar en tareas como la preparación y administración de mates $\mathrm{u}$ otras infusiones, o la realización de ceremonias con valor simbólico para la madre y sus allegados. El personal de salud debe facilitar la entrega de la placenta a la familia para que disponga de ella según las costumbres locales, que a veces suponen su entierro o incineración. De presentarse alguna complicación que amenace la vida de la madre o del recién nacido, se deberá recurrir a un establecimiento de salud de nivel superior.

La norma señala que la atención del parto la realizará el "personal de salud de acuerdo a sus competencias y funciones asignadas", que estará compuesto por médicos, obstetras y personal de enfermería. No considera como personal de salud a las parteras o parteros tradicionales o a los agentes comunitarios de salud. Sin embargo, de otro documento oficial se desprende que en algunos casos los parteros tradicionales pueden atender partos supervisados por profesionales de salud del Ministerio: "si hay partero o partera tradicional, ellos son los que atienden el parto. Nosotros solo controlamos el riesgo. Es mejor hacer eso, entonces la gente nos acepta" (11).

\section{DISCUSIÓN}

Como podemos apreciar, las propuestas programáticas y de política, así como las evaluaciones y diagnósticos realizados por organismos internacionales como la OPS, abordan aspectos clave de los problemas de salud que afectan a los pueblos indígenas de las Américas. La situación desventajosa en que se encuentran muchos de ellos tiene hondas raíces en procesos históricos de larga data, pero no fue sino hasta hace unos 20 ó 25 años que los organismos multilaterales del sistema interamericano y de las Naciones Unidas comenzaron a abordar en profundidad esa cuestión. La preocupación relativamente reciente por la salud de los pueblos indígenas en esos ámbitos no puede entenderse si no se toman en cuenta las profundas transformaciones de las estructuras socioeconómicas como consecuencia del proceso de globalización, que han ido acompañadas de dinámicas políticas y 
culturales específicas y han repercutido en los niveles locales de organización sociocultural. Esas transformaciones también guardan relación con el surgimiento o la presencia creciente de nuevos movimientos sociales que se articulan y cobran fuerza reivindicando demandas basadas en consideraciones culturales, de etnia, género y orientación sexual, entre otras. En el plano de los debates sobre políticas públicas vemos también que se están modificando las propias concepciones sobre el desarrollo. En los años noventa, estos enfoques, que durante mucho tiempo se habían guiado por criterios puramente economicistas, fueron dando cabida a nuevas perspectivas enraizadas, por una parte, en lo que algunos han denominado "el giro participativo", a partir del cual se valoriza la acción de los actores sociales y su conocimiento local, y, por otra parte, en las críticas a los paradigmas que dejaban de lado la cultura como factor del desarrollo. Tales críticas configuraron un ambiente propicio para lo que se denominó "el giro cultural", en el que se trasladaba la cultura de la periferia al centro, y ello permitió incorporar prácticas, significados y discursos interculturales en los análisis, proyectos y políticas sociales y económicas de los países occidentales (12).

En este escenario, la emergencia de movimientos étnicos, y su fortalecimiento en el contexto de las luchas y reivindicaciones que llevaron adelante en defensa de sus derechos ciudadanos, sociales y económicos, con énfasis en el respeto a sus culturas, habría impulsado a organismos como la OPS/OMS a recoger algunas de las preocupaciones de los pueblos indígenas, en este caso las relacionadas con la salud pública. Cabe señalar, sin embargo, que las demandas indígenas suelen suscitar resistencias en los sistemas político-institucionales de los países, y, por otro lado, a los pueblos originarios les cuesta obtener el apoyo de las élites y de buena parte de la población general, más cercanas ambas a la cultura occidental predominante, de modo que sus exigencias han tenido un avance más bien lento en el interior de los países. Algo distinto sucedió cuando esas demandas llegaron a los organismos de las Naciones Unidas, que paulatinamente las fueron tomando en consideración, discutiendo, y en alguna medida aceptando. Creemos que esto se debió a que esos organismos, sobre todo la OPS para el caso que nos ocupa, mantienen orientaciones sustentadas más en lo técnico que en lo político. En las discusiones sobre temas de salud, que muchas veces se resuelven en el ámbito académico, no siempre se manifiestan las fricciones que surgen cuando se tratan asuntos políticos, económicos, limítrofes o de seguridad.

A principios del decenio de 1990, se discutió en la OPS sobre la salud y las condiciones sanitarias de los pueblos indígenas, al mismo tiempo que en los ámbitos políticos, jurídicos y académicos de la región se planteaban roces y tensiones culturales originados en la globalización, y que se observaba la explosión de reclamos locales por la identidad étnica, junto con la emergencia de la interculturalidad como propuesta ético-política para abordar los problemas que plantean los contextos de diversidad cultural. Claro está que, para poner en práctica las propuestas interculturales, y que no queden reducidas a mera retórica, es menester traducirlas en políticas públicas concretas, tales como iniciativas de "acción afirmativa", "compensatorias" o de "discriminación positiva" (13).

En el Perú, la decisión del sistema sanitario de incorporar el parto vertical no parece haberse originado en reclamos políticos de los actores sociales involucrados (de influencia apenas incipiente en el país en esos años), sino en un intento por alcanzar metas de salud pública y como forma de acción afirmativa surgida del Estado en respuesta a las críticas a los enfoques de desarrollo que dejaban de lado las perspectivas culturales. Estas críticas se veían en gran medida potenciadas por los reclamos identitarios cada vez más frecuentes en los debates políticos y académicos tanto regionales como internacionales.

$\mathrm{Al}$ analizar el discurso sobre políticas de salud y las formulaciones metodológicas y programáticas de la OPS, resulta evidente que el proyecto intercultural ha influido de manera apreciable en las orientaciones de este organismo. Este discurso a menudo va más allá del reconocimiento de las diferencias y del respeto y tolerancia para con las culturas indígenas, y hace especial hincapié en la comunicación, el aprendizaje recíproco y el acercamiento entre los pueblos indígenas y la sociedad occidental. Con el fin de ilustrar mejor este punto, conviene esbozar los principales aspectos del debate sobre ambas propuestas: multiculturalidad e interculturalidad. Para simplificar, diremos que la perspectiva "multicultural" recoge la importancia del reconocimiento y respecto a las demás culturas y de la tolerancia con ellas, mientras que el enfoque "intercultural" propone tomar en cuenta el reconocimiento y el respeto, pero ir más allá, señalando que la tolerancia no basta, y que es preciso reforzar el diálogo y la comunicación entre las culturas (14). Es notorio también en el discurso de la OPS el fuerte acento en la promoción de acciones encaminadas a reducir las disparidades e inequidades, y en la necesidad de considerar la cosmovisión y las creencias de los indígenas. En sus planes de acción se destaca i) la importancia del diálogo entre las autoridades gubernamentales, los pueblos indígenas y la sociedad civil; ii) la introducción del "enfoque intercultural" en el sistema de salud, observando que las intervenciones sanitarias sean "culturalmente apropiadas"; y iii) el establecimiento y organización de procedimientos y mecanismos para recoger y sistematizar el conocimiento y las prácticas indígenas sobre la salud y el bienestar.

Estas propuestas, en alguna medida, han servido de guía para la confección de las políticas de salud intercultural en el Perú. Los conceptos empleados en muchos de los documentos técnicos y normativos del Ministerio de Salud permiten advertir que los organismos del sistema sanitario han adoptado en ciertos niveles el espíritu de las recomendaciones de la OPS. Sin embargo, en el caso peruano se echa en falta el apoyo político y financiero necesario para que los 
programas gubernamentales puedan atender eficazmente los problemas de salud de los indígenas. En suma, estos programas tienen un discurso de orientación intercultural que no deja de lado el aprendizaje recíproco ni el diálogo entre el sistema de salud y los pueblos indígenas; pero, en la práctica, diversas limitaciones del sistema sanitario (y en general, del Estado) han impedido que las políticas tengan el alcance e impacto positivos que cabía esperar sobre los estándares globales de salud colectiva de los pueblos indígenas. No obstante, en el terreno específico de la salud reproductiva, es claro que la atención del parto vertical y los logros de esta iniciativa son avances notables que comienzan a tener repercusiones visibles en los indicadores respectivos. Aunque aún persisten ciertas brechas en el acceso a los servicios de salud reproductiva, las encuestas demográficas y de salud familiar (ENDES, 2000 y 2004-2006) muestran reducciones sostenidas en las tasas de mortalidad neonatal: 13 muertes infantiles por 1000 nacidos vivos durante el primer mes de vida en la última encuesta (2004-2006), comparadas con 18 muertes en promedio en los 5 a 9 años anteriores, y 26 muertes en los 10 a 14 años previos. La tasa de mortalidad infantil rural también descendió, de 60 (en la medición del año 2000) a 36 muertes por 1000 nacidos vivos durante el primer año de vida (2004-2006). Se estima que por complicaciones durante el embarazo, el parto o el puerperio, cada año mueren en el Perú 185 mujeres por 100000 nacidos vivos (una de las cifras más altas en América Latina). No obstante, entre 2004 y 2007 el índice de mortalidad materna presenta una disminución sostenida, sobre todo en las zonas altoandinas y selváticas: 628 muertes maternas en 2004, 596 en 2005, 536 en 2006, y 513 en 2007. Esta disminución de los índices de mortalidad materna y neonatal ha sido asociada con la adecuación cultural de los servicios, la implementación del parto vertical y el trabajo de las casas de espera. El Ministerio así lo subraya en su Memoria 2001-2006, junto con el mejoramiento del acceso de las mujeres a los servicios de salud, principalmente en las zonas rurales.

Por otra parte, más allá de los índices globales, si bien la orientación de la política y ciertos criterios técnicos parecen encaminarse positivamente, conviene seguir de cerca la manera concreta en que la interacción con las comunidades se lleva a la práctica. Existen informes sobre casos de maltrato a mujeres y familias indígenas, discriminación racial, atención de mala calidad en algunas casas de espera y servicios de salud, y cobro de "multas" a mujeres que no asisten a los controles prenatales o que dan a luz en sus domicilios (9). Se ha señalado también que el personal de salud actuaba en algunos casos motivado más por las presiones para cumplir con las metas de atención y la mejora de los indicadores que por la idea de la adecuación cultural (15). Conviene tener en cuenta al respecto el planteamiento que distingue entre la interculturalidad en el nivel macro, en las políticas públicas, y la "microinterculturalidad", referida a las relaciones interpersonales y a las "estructuras culturales y simbólicas"
(16). Algunos informes han señalado reacciones de resistencia y desconfianza por parte de la población rural hacia determinados servicios estatales de salud reproductiva $(9,15)$. Consideramos que ello se debe en parte a la falta de diálogo y de capacitación, pero sobre todo a una mirada cultural que subordina e inferioriza al "Otro" indígena, a sus costumbres y modos de vida.

A fin de lograr avances sostenidos, el Ministerio primero tendría que reconocer y evaluar hasta qué punto los prejuicios raciales y los desencuentros culturales afectan el funcionamiento de los servicios de salud y las relaciones entre la población y los prestadores de asistencia sanitaria, para luego poner en práctica formas eficaces de resolver esos problemas, respetando siempre la diversidad de perspectivas en torno a las maneras de entender la salud y practicar la medicina. Por supuesto, es preciso que el Estado amplíe su visión para considerar que muchos de estos roces y desencuentros tienen sus raíces en las profundas inequidades que atraviesan la sociedad peruana, y que las diferencias de poder material y simbólico se hacen presentes también en el nivel de las interacciones entre los usuarios indígenas y los proveedores de servicios de salud, dando forma a las interacciones mismas, así como a las expectativas y actitudes de quienes participan en ellas.

Un documento publicado por la OPS en 2001 (17), que analiza la experiencia de atención del parto vertical en la provincia de Quispicanchi, departamento de Cuzco, propone un modelo de atención del parto vertical desarrollado por un grupo de especialistas del organismo entonces llamado INMETRA. Ese modelo y la reciente norma técnica del Ministerio poseen elementos en común, con la diferencia de que el primero recomendaba, junto con el aumento de la cobertura del parto institucional, impulsar la atención domiciliaria por parteras tradicionales, a las que otorgaba de esa forma un papel más activo, mientras que la norma del Ministerio no considera el parto domiciliario y tampoco la intervención de parteras tradicionales; lo que sí señala es que estas pueden acompañar a las mujeres en el parto, indicar el empleo de preparaciones o hierbas, y ayudar a sostener a la parturienta en las posiciones propias del parto vertical.

En la actual norma técnica (8), por otro lado, entre los especialistas que participaron en la elaboración o revisión del documento figuran cerca de 90 funcionarios (de la ESN de Salud Sexual y Reproductiva, del Instituto Materno-Perinatal, de direcciones generales y regionales del Ministerio, de la seguridad social, de asociaciones profesionales de Obstetricia y Ginecología y de UNICEF), pero no hay ningún representante de otras dependencias del Ministerio que trabajan en el ámbito de la salud intercultural. No figuran especialistas del Centro Nacional de Salud Intercultural/ESN de Salud de los Pueblos Indígenas ni del Grupo Temático de Salud de los Pueblos Indígenas (DGE). Tampoco funcionarios de la OPS/OMS, de ONG, ni representantes de las organizaciones indígenas.

Cabe mencionar también que los esfuerzos del Ministerio por implementar la atención del parto ver- 
tical se concentran en determinadas zonas del país, fundamentalmente en áreas donde se supone que hay poblaciones indígenas. Solo se registra una casa de espera en la zona norte de Lima, pero no se informa sobre la existencia de estos servicios en otras ciudades de la Costa (salvo en el caso de algunas experiencias fuera del sistema público de salud). Si los sistemas de información sanitaria o demográfica consignaran la pertenencia étnica de la población, como recomiendan los planes de acción de la OPS (18), se podría apreciar que una considerable proporción de la población de Lima tiene, en mayor o menor medida, referentes identitarios que corresponden a la mayoría de las etnias del país, sino a todas. Si la migración del campo a la ciudad es uno de los fenómenos sociales que más ha marcado la historia reciente del Perú, convendría apreciar esta realidad para evaluar la conveniencia de la introducción de servicios de salud culturalmente adecuados también en el contexto urbano o en otras zonas de la Costa. Este es un tema complejo que no trataremos aquí, pero que merece ser investigado con más detenimiento.

\section{CONSIDERACIONES FINALES}

Si tomamos en cuenta elementos del debate entre multiculturalidad e interculturalidad, a primera vista se advierte que la política del Ministerio es "intercultural" en unos aspectos (en lo discursivo y programático) y "multicultural" en otros. Ello se debe sobre todo a las manifiestas barreras culturales y asimetrías que persisten en el micronivel, y también a suponer que las poblaciones indígenas solo se encuentran asentadas en la Sierra y en la Selva, donde habría que establecer los servicios de atención del parto vertical —como efectivamente se está haciendo-; en tanto que las mujeres indígenas de las zonas urbanas y de muchos lugares de la Costa — que no son pocas a pesar de ser invisibles para los estudios demográficos y para los sistemas de información- tendrían que resignarse a contar solo con los servicios de atención del parto horizontal bajo el paradigma de la medicina "moderna". Se daría entonces, en el ámbito de la salud, algo similar a lo que se advierte en el caso de la educación: una "ruralización" de la agenda intercultural (16).

Corresponde señalar que estas consideraciones necesitan ser evaluadas con mayor profundidad debido a que la información al respecto aún es escasa o inexistente; en efecto, no sabemos si para las mujeres indígenas gestantes en Lima y otros centros urbanos de la Costa sería importante o deseable que los servicios de salud consideren la atención del parto vertical o si preferirían adoptar la modalidad horizontal. Tampoco sabemos qué factores intervienen en las preferencias, actitudes o percepciones de las mujeres indígenas que viven en las ciudades o en las zonas rurales de la Costa en relación con los servicios de salud reproductiva. Además, convendría tener en la norma técnica una definición clara sobre si, para el Ministerio, pueden o no las parteras y los parteros tradicionales atender partos en el contexto de los servicios de salud, o si conviene o no que el parto tenga lugar en el ámbito domiciliario. $\mathrm{Al}$ parecer, en la elaboración de la norma técnica no se han atendido debidamente las voces de otros organismos (dependencias sanitarias especializadas en interculturalidad, ONG, OPS/OMS), y de los propios indígenas, cuyos aportes podrían arrojar más luz sobre estos asuntos, contribuyendo así a perfeccionar los actuales estándares de atención del parto vertical.

Otra cuestión importante son los desencuentros culturales entre el personal de salud y los indígenas, que se expresan muchas veces bajo la forma de discriminación; sin embargo, no podríamos afirmar que el Ministerio no aborda este problema. De hecho, el INS está realizando estudios de corte cualitativo para la evaluación de servicios de salud en poblaciones indígenas (19), pero la realidad que muestran algunos informes sobre el maltrato a la población constituye un llamamiento a acciones decididas para prevenir este tipo de situaciones. En todo caso, la OPS y otros organismos, incluidas las ONG, han formulado recomendaciones y elaborado planes $(18,20)$ sobre lo que se puede y se debe hacer para afrontar los problemas relacionados con las diferencias culturales.

Finalmente, conviene matizar las críticas vertidas aquí señalando, en primer lugar, que hay que aplaudir tanto la preocupación del Ministerio por articular políticas interculturales como los logros de la iniciativa de atención del parto vertical. En segundo lugar, y teniendo en cuenta que la incorporación del enfoque intercultural en salud es relativamente reciente, cabría esperar que los ajustes que puedan parecer necesarios a las políticas y programas de salud en este campo comiencen a ser implementados como producto del aprendizaje que en el camino y con la experiencia vayan alcanzando todos los actores involucrados.

\section{SYNOPSIS}

\section{Incorporation of an intercultural approach in the Peruvian health care system: the vertical birth method}

Peru has a large indigenous population (40\% of the total), made of 72 distinct groups. These peoples are at a great disadvantage compared to the mestizos and other groups that are more closely connected with the dominant culture. The political and economic inequality and inequities are particularly stark with regard to health conditions. National policy and plans have not considered the indigenous peoples' values and identity, nor their right to self-determination and control of their land and resources, and even less so, their health knowledge and practices, inasmuch western "modern medicine" has been imposed upon them by the dominant culture.

Since 1992, as a result of international forums and academia, as well as a growing movement in Peru to acknowledge the value of cultural diversity and the rights of indigenous peoples, Government proposals, initiatives, and 
programs were undertaken to incorporate and empower cultural contributions and traditional practices in a way that would not only benefit the indigenous, but also enrich, through various intercultural venues, the stock of cultural traditions and ethnic roots of the greater Peruvian society.

This article explores specifically how these intercultural strategies have been rolled out in health care to institutionalize the vertical birth method in rural, predominantly in- digenous, communities, and endeavors to assess the decrease in maternal mortality, as well as the difficulties encountered, and mostly overcome, by expressing multiculturalism in the health field.

Key words: Cultural diversity; birth; indigenous health; health policy; Peru.

\section{REFERENCIAS}

1. Perú, Ministerio de Salud. La heterogeneidad etnolingüística del Perú: situación socioeconómica y de salud de la población indígena. Lima: MINSA; 1999.

2. Instituto Indigenista Peruano (IIP) Mapa etnolingüístico oficial del Perú. Lima: Ministerio de Agricultura; 1994.

3. Organización Panamericana de la Salud. La salud de los pueblos indígenas de las Américas. [138.a Sesión del Comité Ejecutivo. CE138/13. Washington, D.C., 12 de junio de 2006] Washing ton, D.C.: OPS; 2006.

4. Fraser B. Providing medical care in the Peruvian Amazon. Lancet. 2006;386: 1408-1409.

5. Perú, Ministerio de Salud. Análisis de situación de salud del pueblo Matsigenka. Lima: MINSA; 2006.

6. Perú, Ministerio de Salud. Análisis de la situación de salud del pueblo ShipiboKonibo. Lima: MINSA; 2002.

7. UNICEF Lima, Instituto Nacional de Estadística e Informática. El estado de la niñez en el Perú. Lima: UNICEF, INEI, 2008.

8. Perú, Ministerio de Salud. Norma técnica para la atención del parto vertical con adecuación intercultural. Lima: MINSA; 2005.

9. Perú, Ministerio de Salud, Proyecto 2000. Salvarse con bien: el parto de la vida en los Andes y la Amazonía del Perú. Lima: MINSA; 1999.
10. Galarza C, Aguilar M, Flores F. Participación de agentes comunitarios de salud para incrementar el control prenatal y la atención del parto institucional en la UTES Huanta. En: Perú, Ministerio de Salud, Proyecto 2000. Investigaciones operativas en salud materno infantil. Lima: MINSA; 1999. Pp. 13-23.

11. Perú, Ministerio de Salud. Carta de la Salud: escuchando la voz de la población. Lima: MINSA/CARE Perú; 2006.

12. Degregori CI, Huber L. Cultura, poder y desarrollo rural. En: Iguíñiz J, Escobal J, Degregori $\mathrm{CI}$, eds. Perú: el problema agrario en debate. SEPIA XI. Lima: SEPIA; 2006. Pp. 452-500.

13. Taylor C. El multiculturalismo y la política del reconocimiento. México, D.F.: Fondo de Cultura Económica; 1993.

14. Degregori CI. Multiculturalidad e interculturalidad. En: Educación y diversidad rural, Seminario Taller, julio de 1998. Lima: Ministerio de Educación; 1999. Pp. 63-69.

15. Bedriñana E, Loayza E. Proyecto: Provisión de servicios de salud sexual y reproductiva culturalmente adecuados en comunidades afectadas por la violencia en Ayacucho. Informe de investigación. Ayacucho: Salud Sin Límites; 2000.

16. Tubino F. Entre el multiculturalismo y la interculturalidad: más allá de la discriminación positiva. En: Fuller N., ed. Interculturalidad y política: desafíos y posibilidades. Lima: Red para el Desarrollo de las Ciencias Sociales; 2002. Pp. 51-76.

17. Brocker A, Sánchez G, Arévalo M, Espinoza G. Promoción de la medicina y prácticas indígenas en la atención primaria de salud: el caso de los quechua del Perú. Atención del parto con la articulación de la medicina tradicional y la medicina académica. Provincia de Quispicanchi, Departamento de Cuzco, Perú. Washington, D.C.: OPS; 2001.

18. Rojas R. Programa Salud de los Pueblos Indígenas de las Américas: plan de acción 2005-2007. Washington, D.C.: OPS; 2004.

19. Quispe R, Córdova R. Método cualitativo con enfoque intercultural - MCEI, herramienta para evaluar servicios de salud en población indígena andina. Bol Instit Nac Salud (Perú). 2005;11/12: 302-305.

20. Salud Sin Límites. Modelo de atención de parto con adecuación intercultural: sistematización de experiencias innovadoras de lucha contra la pobreza rural en la Región Andina. s.c.: Salud Sin Límites; 2004.

Manuscrito recibido el 28 de diciembre de 2007 Aceptado para publicación, tras revisión, el 31 de agosto de 2008. 